\title{
WEAK $C^{*}$-HOPF ALGEBRAS: THE COASSOCIATIVE SYMMETRY OF NON-INTEGRAL DIMENSIONS
}

\author{
GABRIELLA BÖHM and KORNÉL SZLACHÁNYI \\ Central Research Institute for Physics \\ H-1525 Budapest 114, P.O.B. 49, Hungary \\ E-mail:BGABR@rmki.kfki.hu,SZLACH@rmki.kfki.hu
}

\begin{abstract}
By allowing the coproduct to be non-unital and weakening the counit and antipode axioms of a $C^{*}$-Hopf algebra too, we obtain a selfdual set of axioms describing a coassociative quantum group, that we call a weak $C^{*}-H o p f$ algebra, which is sufficiently general to describe the symmetries of essentially arbitrary fusion rules. It is the same structure that can be obtained by replacing the multiplicative unitary of Baaj and Skandalis with a partial isometry. The algebraic properties, the existence of the Haar measure and representation theory are briefly discussed. An algorithm is explained how to construct examples (in particular ones with non-integral dimensions) from non-Abelian cohomology.
\end{abstract}

1. Introduction. Recently the notion of weak $C^{*}$-Hopf algebra has been proposed [3] to describe the symmetry underlying a (strict) monoidal compact $C^{*}$-category generated by finitely many irreducible objects. Finite dimensional weak $C^{*}$-Hopf algebras are the proper generalizations of finite groups which cover also the situations where the intrinsic dimensions $d_{q}$ of some irreducible representations have non-integral values. Weak Hopf algebras provide an axiomatic approach corresponding to - and in a certain sense generalizing - the combinatorial concept of 'quantum groupoids' proposed by A. Ocneanu [12]. In contrast to weak quasi Hopf algebras [10] and rational Hopf algebras [16], weak Hopf algebras have the advantage of being coassociative. This allows one to define actions of the symmetry and leads to the notion of a crossed product algebra within the $C^{*}$-algebra framework. The price one has to pay for having coassociativity and nonintegral dimensions at the same time is that the counits of weak Hopf algebras are not

Research of the first author supported by the Hungarian Scientific Research Fund, OTKA T 016233.

Research of the second author supported by the Hungarian Scientific Research Fund, OTKA1815.

1991 Mathematics Subject Classification: Primary: 81R50, Secondary: 57T05, 17B37.

The paper is in final form and no version of it will be published elsewhere. 
algebra maps (weak Hopf algebras do not have 1-dimensional representations) in general. The notion of the weak Hopf algebra seems to provide the appropriate symmetry concept for finite index reducible depth 2 inclusions of factors [11]. It may be important also to the problem of quantum symmetry in low dimensional quantum field theories $[6,13,14]$ in the sense of the Doplicher-Roberts theory [4].

The way a weak Hopf algebra manages to remain coassociative while having a representation category equivalent to that of a relaxed monoidal category can be visualized by the "blowing up" procedure. Let $H$ be a quasi-Hopf algebra and define $A=H \otimes M_{n}$. The irreducible representations of $H$ are clearly in one-to-one correspondence with those of the blown up algebra $A$. Now the point is that there is more flexibility in skrooching the coproduct on $A$ than in $H$. Thus one may expect that if $n$ is large enough an appropriate skrooching will turn the coproduct on $A$ to be coassociative. This was explicitely checked on the example of the quasi-double of a finite group in [3].

We do not claim, however, that every weak Hopf algebra arises as a blowing up of a (weak) quasi-Hopf algebra. In fact the interesting cases are the ones with non-integral dimensions and these never arise in this way.

In Sect. 2 we briefly review the main properties of weak Hopf algebras in the axiomatic approach, without proofs. Namely: we consider the definition of the dual of a finite dimensional weak $C^{*}$-Hopf algebra, the representation theory and the existence of a Haar integral. The details will be published in [1].

The characteristic feature distinguishing a weak $C^{*}$-Hopf algebra $A$ from a Hopf algebra is the occurence of certain sub- $C^{*}$-algebras $A^{L}$ and $A^{R}$ within $A$ that behave like non-commutative generalizations of the number field $\mathbb{C}$ in many senses. For example, every representation of a pure weak $C^{*}$-Hopf algebra $A$ represents $A^{L}$ and $A^{R}$ faithfully. Or, the crossed product $M \rtimes A$ of a $C^{*}$-algebra $M$ with respect to a left action of $A$ on $M$ is an amalgamated tensor product $M \otimes_{A^{L}} A$ where $A^{L}$ gets identified with an appropriate subalgebra in $M$. In the Weyl algebra $\hat{A} \rtimes A$ this subalgebra is just $\hat{A}^{R}$. There is a generalization of Drinfeld's quantum double $A \bowtie \hat{A}$ [5] for a weak $C^{*}$-Hopf algebra $A$ which, as a linear space, turns out to be the amalgamated tensor product of $A$ and $\hat{A}$ with the identifications $A^{R} \cong \hat{A}^{L}$ and $A^{L} \cong \hat{A}^{R}$ ('cyclic' amalgamation). This double has again the structure of a weak $C^{*}$-Hopf algebra.

In Sect.3 we discuss Ocneanu's quantum cohomology [12], which allows one to construct a weak Hopf algebra from the data $(K, F)$ where $K$ is a finite simplicial complex having only two vertices $\bullet$ and $\circ$ and $F$ is an appropriate 3-cocycle on $K$. A subcomplex $K_{\bullet}$ of $K$ and the restriction $F_{0}=\left.F\right|_{K_{\bullet}}$ form the data $\left(K_{\bullet}, F_{0}\right)$ determined up to equivalence uniquely by a monoidal category $\mathcal{C}$. The edges of $K$ • correspond to irreducible objects $r$, the faces to intertwiners $T: r \rightarrow p \times q$, and the values of $F_{0}$ on the tetrahedra to the recoupling coefficients of $\mathcal{C}$. There exist several possible ways to extend the data $\left(K_{\bullet}, F_{0}\right)$ to $(K, F)$, but each such extension determines a unique weak $C^{*}$-Hopf algebra $A$ the 6 -j symbols of which are given precisely by $F_{0}$. Among the various possibilities there exists a "canonical" one which assigns to $\mathcal{C}$ a selfdual weak $C^{*}$-Hopf algebra $A \cong \hat{A}$ as its underlying symmetry algebra. 
Weak Hopf algebras are also related to a generalization of the multiplicative unitary of [2]. Let $\mathcal{H}$ be a finite dimensional Hilbert space and $V$ be a partial isometry on $\mathcal{H} \otimes \mathcal{H}$. We call $V$ a multiplicative isometry if the following relations hold on $\mathcal{H} \otimes \mathcal{H} \otimes \mathcal{H}$ :

$$
\begin{array}{ll}
V_{12} V_{13} V_{23}=V_{23} V_{12} & V_{13} V_{23} V_{23}^{*}=V_{12}^{*} V_{12} V_{13} \\
V_{23}^{*} V_{12} V_{13}=V_{12} V_{23}^{*} & V_{12} V_{12}^{*} V_{23}=V_{23} V_{12} V_{12}^{*}
\end{array}
$$

If $A$ is a weak $C^{*}$-Hopf algebra with a basis $\left\{b^{i}\right\}$ and $\left\{\beta_{i}\right\}$ is the dual basis in $\hat{A}$ then $V=\sum_{i} \beta_{i} \otimes b^{i}$ becomes a multiplicative isometry in any representation of the Weyl algebra $A \rtimes \hat{A}$. Under certain regularity conditions on $V$ this is the most general example of a multiplicative isometry and the weak $C^{*}$-Hopf algebra $A$ can be uniquely reconstructed from $V$. We wish to discuss this problem in a subsequent publication.

2. Weak $C^{*}$-Hopf algebras. In the sequel we will use Sweedler's notation [15]: For a coproduct $\Delta: A \rightarrow A \otimes A$ we write $\Delta(x)=x_{(1)} \otimes x_{(2)}$. Using coassociativity the iterated coproduct $x_{(1)} \otimes x_{(2)(1)} \otimes x_{(2)(2)}$ can be simply written as $x_{(1)} \otimes x_{(2)} \otimes x_{(3)}$.

A weak ${ }^{*}$-Hopf algebra is a ${ }^{*}$-algebra $A$ with unit $\mathbb{1 1}$ together with linear maps $\Delta: A \rightarrow$ $A \otimes A, \varepsilon: A \rightarrow \mathbb{C}$, and $S: A \rightarrow A$ called the coproduct, the counit, and the antipode respectively, if the following axioms hold:

$$
\begin{aligned}
\Delta(x y) & =\Delta(x) \Delta(y) \\
\Delta\left(x^{*}\right) & =\Delta(x)^{*} \\
(\Delta \otimes i d) \circ \Delta & =(i d \otimes \Delta) \circ \Delta \\
\varepsilon(x y) & =\varepsilon\left(x \mathbb{1}_{(1)}\right) \varepsilon\left(\mathbb{1}_{(2)} y\right) \\
\varepsilon\left(x^{*} x\right) & \geq 0 \\
(\varepsilon \otimes i d) \circ \Delta= & i d=(i d \otimes \varepsilon) \circ \Delta \\
S(x y) & =S(y) S(x) \\
S \circ^{*} \circ S \circ^{*} & =i d \\
\Delta \circ S & =(S \otimes S) \circ \Delta^{o p} \\
S\left(x_{(1)}\right) x_{(2)} \otimes x_{(3)} & =\mathbb{1}_{(1)} \otimes x \mathbb{1}_{(2)}
\end{aligned}
$$

for all $x, y \in A$.

The weak *-algebra $A$ is quasitriangular if there exists an element $R \in A \otimes A$ such that

$$
\begin{array}{rll}
R \Delta(x) \quad= & \Delta^{o p}(x) R \\
R R^{*}=\Delta^{o p}(\mathbb{1 1}) & & R^{*} R=\Delta(\mathbb{1}) \\
(i d \otimes \Delta)(R)=R_{13} R_{12} & & (\Delta \otimes i d)(R)=R_{13} R_{23}
\end{array}
$$

If furthermore $A$ possesses a faithful *-representation on a Hilbert space it is called a weak $C^{*}$-Hopf algebra.

In the case when $A$ is a finite dimensional $C^{*}$ algebra the axiom $(A .2 b)$ may be replaced with the apparently weaker requirement that $\varepsilon$ be ${ }^{*}$-preserving: $\varepsilon 0^{*}={ }^{-} \circ \varepsilon$. 
( ${ }^{-}$means complex conjugation.) The resulting set of axioms is then equivalent to $(A .1-4)$ above.

In order to recover the axioms (and some of their consequences) of a $(C)^{*}$-Hopf algebra, we can add either the condition that the coproduct be unital or that $\varepsilon$ be multiplicative or that the maps $\Pi^{L / R}$ defined by

$$
\Pi^{L}(x):=x_{(1)} S\left(x_{(2)}\right), \quad \Pi^{R}(x):=S\left(x_{(1)}\right) x_{(2)}
$$

both reduce to $\mathbb{1} \varepsilon(x)$. In our more general case these maps - though are not conditional expectations- are linear projections onto nontrivial ${ }^{*}$-subalgebras $A^{L}$ and $A^{R}$, respectively, that are isomorphic to each other. $A^{L}$ lies in the relative commutant of $A^{R}$. The antipode $S$ maps them onto each other. The restriction of $\frac{1}{\varepsilon(1)} \varepsilon$ is a faithful state both on $A^{L}$ and $A^{R}$, so these subalgebras are faithfully represented in the GNS representation corresponding to this state, called the trivial representation. One also proves that $\Delta(\mathbb{1})$ lies in $A^{R} \otimes A^{L}$.

The dual $\hat{A}$ of $A$ is defined to be the space of linear functionals $\varphi$ on $A$ and is equipped with a multiplication and a comultiplication obtained by tranposing the comultiplication and multiplication of $A$ w.r.t the canonical pairing $\langle\rangle:, \hat{A} \times A \rightarrow \mathbb{C}$. The unit element of $\hat{A}$ is $\hat{\mathbb{1}}:=\varepsilon$. The antipode $\hat{S}$ and the ${ }^{*}$-operation of $\hat{A}$ are defined respectively by

$$
\begin{aligned}
\langle\hat{S}(\varphi), x\rangle & =\langle\varphi, S(x)\rangle \\
\left\langle\varphi^{*}, x\right\rangle & =\frac{\left\langle\varphi, S(x)^{*}\right\rangle}{\langle\varphi}
\end{aligned}
$$

It is proven in [3] that if $(A, \mathbb{1}, \Delta, \varepsilon, S)$ satisfies Axioms (A1-4) and $\operatorname{dim} A$ is finite then $(\hat{A}, \hat{\mathbb{1}}, \hat{\Delta}, \hat{\varepsilon}, \hat{S})$ satisfies Axioms $(\mathrm{A} 1-4)$, too. That is the notion of a finite dimensional weak *-Hopf algebra is selfdual.

The representation theory of a finite dimensional weak $C^{*}$-Hopf algebra $A$ generalizes the one of a finite group (i.e it is a 'finite quantum group') in the sense that the category $\mathcal{R} \operatorname{ep} A$, whose objects are the finite dimensional (not necessarily non-degenerate) *-representations of $A$ and the arrows the intertwiners between them is a compact monoidal $C^{*}$-category. If $A$ is quasitriangular then $\mathcal{R} e p A$ is braided.

An interesting phenomenon of the theory of finite dimensional weak $C^{*}$-Hopf algebras is that their trivial representation may be reducible. More precisely: the irreducible objects of $\mathcal{R} e p A$ are of two different kinds. There are nilpotent ones, that is for which $D \times D$ is the zero object. The irreducible objects that are not nilpotent generate a monoidal full subcategory $\mathcal{R} e p_{0} A$ with conjugates. Furthermore the set of objects of $\mathcal{R} e p_{0} A$ has a unique partition into subsets, such that the elements of each subset define a monoidal full subcategory with conjugates, and the monoidal unit in each subcategory is irreducible. The monoidal product of objects that belong to different subcategories is the zero object, and the intertwiner space between them is 0 dimensional.

If the fusion rules of $A$ are Abelian then there are no nilpotent representations. In the language of $C^{*}$ algebras the partition of the irreducible representations corresponds to a decomposition into a direct sum of $C^{*}$-algebras. That is $A$ is a direct sum of two $C^{*}$ algebras: the 'semisimple' and the 'nilpotent' part. The 'semisimple' part can be decomposed as a weak $C^{*}$-Hopf algebra into a direct sum of pure weak $C^{*}$-Hopf algebras, that is to ones on which the state $\frac{1}{\varepsilon(1)} \varepsilon$ is pure (hence the trivial representation irreducible). 
Like compact groups finite dimensional weak $C^{*}$-Hopf algebras possess unique Haar measures in the following sense. There exists a unique $h \in A$ characterized by the property

$$
x h=\Pi^{L}(x) h, \quad h x=h \Pi^{R}(x), \quad \forall x \in A .
$$

and by the normalization conditions $\Pi^{L}(h)=\mathbb{1}=\Pi^{R}(h)$. For Hopf algebras $\Pi^{L / R}=\mathbb{1} \varepsilon$, therefore this definition coincides with the usual one [15]. The Haar measure $h$ satisfies the following important properties: it is a hermitean projector invariant under $S$ and

$$
\begin{aligned}
\langle\hat{\mathbb{1}}, h\rangle & =\varepsilon(\mathbb{1}) \\
h_{(1)} x \otimes h_{(2)} & =h_{(1)} \otimes h_{(2)} S(x) \quad x \in A
\end{aligned}
$$

One proves that the Haar measure defines a faithful state on the dual via the formula $\hat{A} \ni \varphi \mapsto\langle\varphi, h\rangle$, so $\hat{A}$ is also a $C^{*}$-algebra, proving that the dual of a finite dimensional weak $C^{*}$-Hopf algebra is a weak $C^{*}$-Hopf algebra again.

A new feature compared to Hopf algebras is that since $S^{2} \neq i d$, similarly to Woronowicz's compact matrix pseudogroups [17], the Haar state is not a trace in general.

3. Examples via non-abelian cohomology. In this Section we give an algorithm, to construct a pair of finite dimensional weak $C^{*}$-Hopf algebras in duality from a solution of a pentagon type of equation. Similar situation is met in Algebraic Quantum Field Theory[8], when one is going to reconstruct the superselection symmetry of a given model. As it was pointed out in [9], the field theoretical model determines some representation theoretical data of the underlying symmetry, such as the fusion coefficients $N_{p q}^{r}$, the recoupling coefficients $F$ and the braiding matrices, satisfying some polynomial (pentagonal and hexagonal) equations. (The braiding aspect - so the hexagon equation - is not treated here.) Although our construction requires more than what is provided by the data $N_{p q}^{r}$ and $F$, it is always possible to extend them in such a way that they determine a weak $C^{*}$-Hopf algebra.

First we describe in detail the input of our construction: a simplicial complex $K$ with a $\mathbb{C}$-valued 3 -chain $F$, satisfying a unitarity condition and the Big Pentagon equation.

The complex $K$ has two 0 -simplices, - and $\bigcirc$ and three kinds of 1-simplices: $\bigcirc \stackrel{i}{\longrightarrow} \mathrm{O}, \stackrel{j}{\longrightarrow} \bullet$, and $\bigcirc \stackrel{k}{\longrightarrow}$, where $i, j$ and $k$ run through three possibly different finite index sets. The 2 -simplices are built up from the allowed 1-simplices:

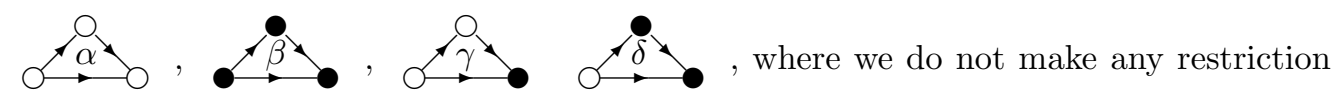

on the finite index sets run through by $\alpha, \beta, \gamma$ and $\delta$. The 3-simplices are built up from the 2 -simplices and they carry no new index:
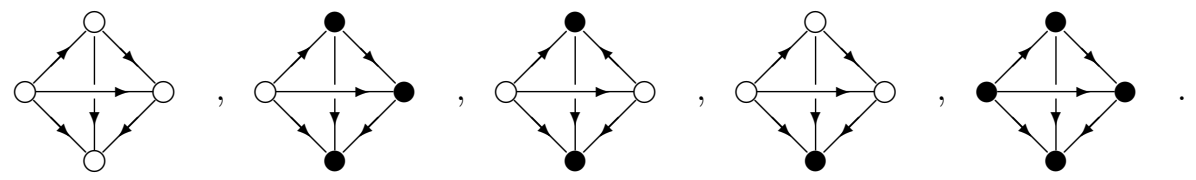

Let $F$ be a $\mathbb{C}$-valued 3 -chain on this complex. We call its $k^{\prime}$ th component its restriction to the 3 -simplices with $k \circ$ and $4-k$

vertices. We use the following index-notation 
for any component of $F$ :

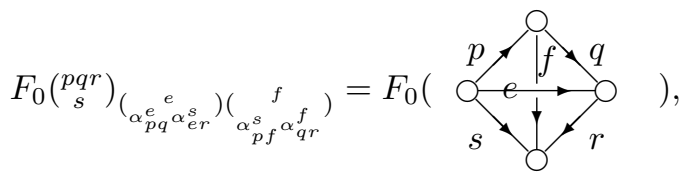

where $\alpha_{p q}^{e}$ is the face index of the 2-simplex components $F_{k}$ is unitary in the sense that

$$
\begin{aligned}
& \sum_{e \alpha \beta}{\overline{F_{k}\left(\begin{array}{c}
p q r \\
s
\end{array}\right)}}_{\left(\begin{array}{c}
e \\
\alpha \beta
\end{array}\right)\left(\begin{array}{c}
f \\
\gamma \delta
\end{array}\right)} F_{k}\left(\begin{array}{c}
p q r \\
s
\end{array}\right)_{\left(\begin{array}{c}
e \\
\alpha \beta
\end{array}\right)\left(\begin{array}{l}
\gamma^{\prime} \delta^{\prime} \\
f^{\prime}
\end{array}\right)}=\delta_{f f^{\prime}} \delta_{\gamma \gamma^{\prime}} \delta_{\delta, \delta^{\prime}}
\end{aligned}
$$

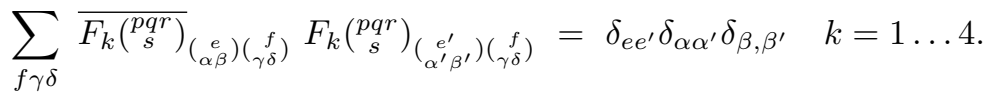

Then $F_{2}$ is invertible in the sense that there exists another 3-chain $\tilde{F}_{2}$ supported on the same 3 -simplices as $F_{2}$, such that

$$
\sum_{r \beta \delta} \tilde{F}_{2}\left(\begin{array}{c}
p q r \\
s
\end{array}\right)_{\left(\begin{array}{c}
e \\
\alpha \beta
\end{array}\right)\left(\begin{array}{c}
f \\
\gamma \delta
\end{array}\right)} F_{2}\left(\begin{array}{c}
p^{\prime} q r \\
s
\end{array}\right)_{\left(\begin{array}{c}
\alpha^{\prime} \beta \\
\gamma_{\gamma^{\prime} \delta}
\end{array}\right)}^{f}=\delta_{p p^{\prime}} \delta_{\alpha, \alpha^{\prime}}, \delta_{\gamma \gamma^{\prime}}
$$

$F$ is called a 3 -cocycle if it satisfies the Big Pentagon equation expressing the requirement that the value of $F$ computed in the two triangularizations of the object

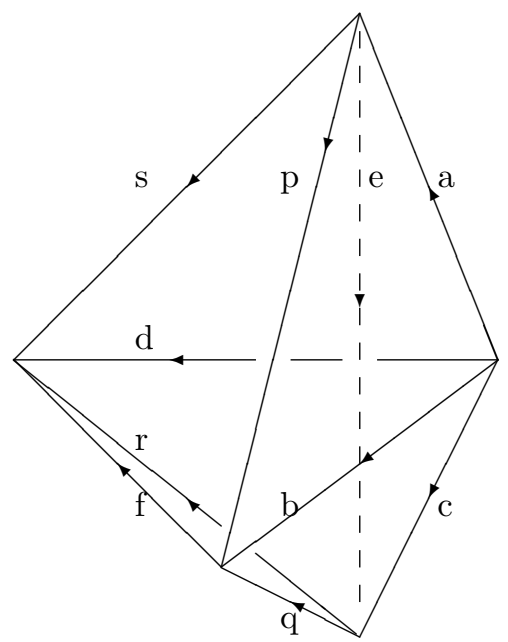

(with any of its allowed colorings) be the same. Explicitly:

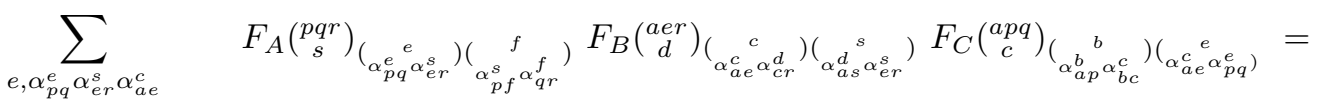

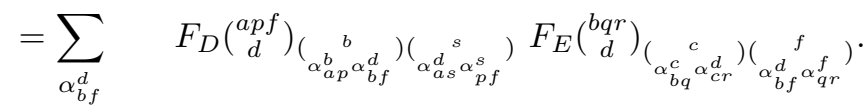


Depending on the values of $\{A B C D E\}$ (the coloring of the figure) we call them $(P 0)$ (for $\{00000\}),(P 1)$ (for $\{01111\}),(P 2)$ (for $\{12221\}),(P 3)$ (for $\{22332\},(P 4)$ (for $\{33433\}$ ) and $(P 5)$ (for $\{44444\})$. (In any case the name $(P k)$ refers to the number $k$ of the appearing vertices of type $\bigcirc$.) These equations are not all independent: $(P 0)$ and $(P 5)$ do follow from the others.

In the Quantum Field Theory context we are given only the subcomplex $K_{\bullet}$ and the restriction $F_{0}$ of the 3 -cocycle on it. We now describe a "canonical" way to extend these information to complete the data $(K, F)$. The complex is defined as follows. Its 1-simplices with any allowed coloring are labelled with the irreducible sectors (charges) $i, j, k, \ldots$ of the model. The 2-simplices

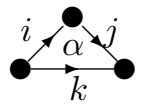
(and of any other allowed coloring) carry indices $\alpha=1 \ldots N_{i j}^{k}$, where $N_{i j}^{k}$ are the fusion coefficients determined by the field theoretical data. The components of the solution of the Big Pentagon equation also coincide, that is we have $F_{0}=F_{1}=F_{2}=F_{3}=F_{4}$ and are equal to the recoupling coefficients obtained from the physical model.

Having a complex $K$ and a unitary solution $F$ of the Big Pentagon equation on it we can define two finite dimensional weak $C^{*}$-Hopf algebras in duality: $A$ corresponding to the vertex $\bullet$ and $\hat{A}$ corresponding to $\bigcirc$. As a $C^{*}$-algebra $A$ is a direct sum for the edges $\longrightarrow$ ? $: A:=\oplus_{q}$ End $V_{q}$, where $V_{q}$ is defined to be the Hilbert space spanned by the orhtonormal basis

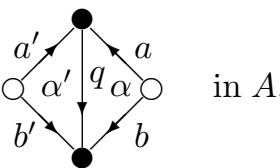

The $C^{*}$ algebra $\hat{A}$ is defined just the same way but the role of $\bullet$ and $\bigcirc$ interchanged: it has the matrix units help of $\tilde{F}_{2}$. Computed it on two matrix units, the result is $\tilde{F}_{2}$ evaluated on the terahedron obtained by rotating the square in the first argument by $180^{\circ}$ around its 'main diagonal' and glued together with the square in the second argument at their coinciding edges:

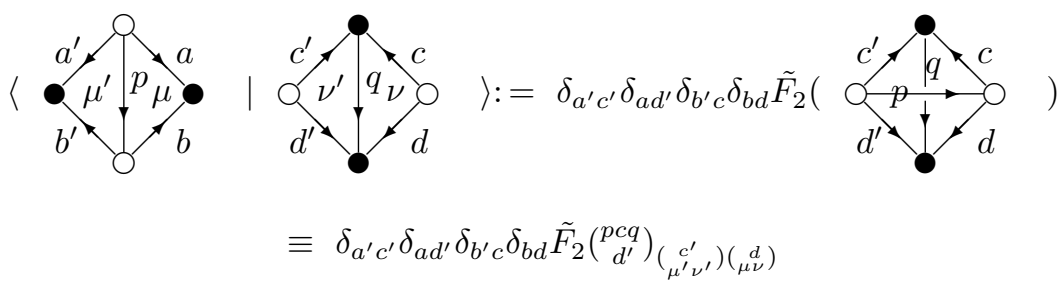

We can then define the comultiplication on $A$ as the transpose of the multiplication in $\hat{A}$, the counit as the pairing with the unit of $\hat{A}$. The antipode $S$ is defined with the formula: $\overline{\left\langle\phi^{*} \mid x^{*}\right\rangle}:=\left\langle\phi \mid S^{-1}(x)\right\rangle$. ( ${ }^{-}$means complex conjugation.) The weak Hopf algebra structre of $\hat{A}$ is the transpose of the *-algebra structure of $A$ in the same way. Our definition then 
implies that the copruduts are coassociative $((A .1 c))$, the counits are coalgebraic counits $((A .2 c))$ and $S \circ^{*} \circ S \circ^{*}=i d((A .3 c))$ in both cases. The remaining axioms are verified using the concrete forms of the coproducts (computed using $(P 2)$ and $(P 3)$, respectively)

$$
=\sum_{p q} \sum_{x^{\prime} x} \sum_{\mu^{\prime} \mu \kappa^{\prime} \kappa \lambda} \sum_{x^{\prime} x}
$$

and of the antipodes

$$
\begin{aligned}
& S\left(\underset{\nu^{\prime}}{\nu_{\nu}^{\prime}}\right)=\sum_{p q^{\prime}} \sum_{\kappa^{\prime} \kappa \mu^{\prime} \mu} \\
& \hat{S}\left(\stackrel{\nu^{\prime} q_{\nu}}{a}\right)=\sum_{p q^{\prime}} \sum_{\kappa^{\prime} \kappa \mu^{\prime} \mu}
\end{aligned}
$$

Axiom $(A .1 b)$ then trivially holds both in $A$ and $\hat{A}$. This implies that so do $(A .3 a)$ and $(A .3 b)$. (A.1a) follows from the unitarity of $F_{1}$. Instead of axiom $(A .2 a)$ for $A$ we can prove using the unitarity of $F_{3}$ the stronger statement that $\hat{\mathbb{1}}_{(1)} \otimes \hat{\mathbb{1}}_{(2)} \hat{\mathbb{1}}_{(1)^{\prime}} \otimes \hat{\mathbb{1}}_{(2)^{\prime}}=$ $\hat{\mathbb{1}}_{(1)} \otimes \hat{\mathbb{1}}_{(2)} \otimes \hat{\mathbb{1}}_{(3)}$ in $\hat{A}$. According to our remark after Axioms $(A .1-4),(A .2 b)$ is now equivalent to the the ${ }^{*}$-preserving property of the counit, which trivially holds. The proof of (A.4) needs some calculation using (3.7) and (3.8), (P2) and the unitarity of $F_{2}$ and $F_{1}$. This proves that $A$ is a weak $C^{*}$-Hopf algebra, and by our result about the duals so is $\hat{A}$.

The components of $F$ have representation theoretical meaning: If $p, q$ and $r$ label 1-simplices of type $\longrightarrow$ - then the fusion coefficient $N_{p q}^{r}$ for $A$ is the number of the 
2-simplices

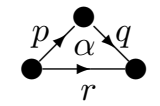

so we may choose $\alpha=1 \ldots N_{p q}^{r}$. In the basis

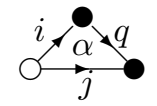

the

Wigner coefficients $T_{p q}^{r \alpha}$ of $A$ have the matrix elements

$$
\left(T_{p q}^{r \alpha}\right)_{(i \gamma k)(l \delta j)}^{(m \beta n)}=\delta_{k l} \delta_{i m} \delta_{j n} F_{1}\left(\begin{array}{c}
i p q \\
j
\end{array}\right){ }_{\left(\begin{array}{c}
k \\
\gamma \delta
\end{array}\right)\left(\begin{array}{c}
r \\
\beta \alpha
\end{array}\right)}
$$

$F_{0}$ then comprises the corresponding recoupling coefficients (see $(P 1)$ ). Similarly, the fusion coefficients for $\hat{A}$ are equal to the number of 2 -simplices of type

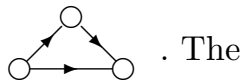
matrix elements of the Wigner coefficients in the basis

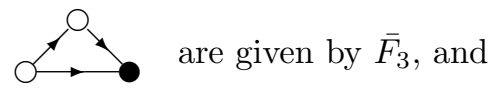
the recoupling coefficients by $\bar{F}_{4}$.

Let us illustrate the above construction on two examples. The first one describes the Lee-Yang fusion rules: $N_{00}^{0}=1, N_{01}^{1}=N_{10}^{1}=1$ and $N_{11}^{0}=N_{11}^{1}=1$, otherwise $N_{i j}^{k}=0$. The complex is defined to have the 0 -simplices $\bullet$ and $\bigcirc$. The 1 -simplices of the kind $\stackrel{i}{\longrightarrow} \mathrm{O}$ and $i$ are all labelled with $i=0,1$. We have the 2-simplices
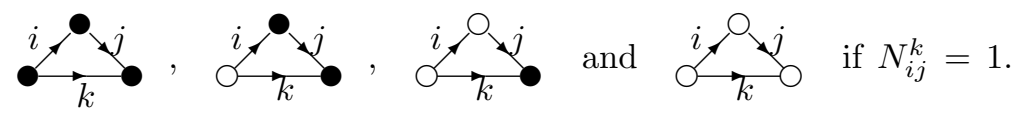

face-indices are introduced.) The 3-simplices are built up from the 2-simplices whithout any further constraint. The solution of $(P 0)$ on $K_{\bullet}$ can be found in [7]:

$$
\begin{aligned}
& F\left(\begin{array}{c}
000 \\
0
\end{array}\right)_{00}=F\left(\begin{array}{c}
011 \\
0
\end{array}\right)_{10}=F\left(\begin{array}{c}
001 \\
1
\end{array}\right)_{01}=F\left(\begin{array}{c}
010 \\
1
\end{array}\right)_{11}=F\left(\begin{array}{c}
011 \\
1
\end{array}\right)_{11}=F\left(\begin{array}{c}
110 \\
0
\end{array}\right)_{01}= \\
& F\left(\begin{array}{c}
111 \\
1
\end{array}\right)_{10}=F\left(\begin{array}{c}
101 \\
0
\end{array}\right)_{11}=F\left(\begin{array}{c}
111 \\
0
\end{array}\right)_{11}=F\left(\begin{array}{c}
100 \\
1
\end{array}\right)_{10}=F\left(\begin{array}{c}
101 \\
1
\end{array}\right)_{11}=F\left(\begin{array}{c}
110 \\
1
\end{array}\right)_{11}=1 \\
& F\left(\begin{array}{c}
111 \\
1
\end{array}\right)_{00}=-F\left(\begin{array}{c}
111 \\
1
\end{array}\right)_{11}=z^{2}
\end{aligned}
$$

where $z=\sqrt{\frac{\sqrt{5}-1}{2}}$. It can be extended to a solution of the Big Pentagon equation by $F_{1}=F_{2}=F_{3}=F_{4}:=F_{0}$. The resulting weak $C^{*}$-Hopf algebra structure (leading to non-integral dimensions) is described in [3].

The other example describes the "blowing up" of a finite group $G$. As a $C^{*}$-algebra $A$ is $C(G) \otimes M_{|G|}(\mathbb{C})$ where $C(G)$ denotes the Abelian algebra of complex valued functions on $G$. The corresponding complex has the 0 -simplices $\bullet$ and $\bigcirc$. All the 1 -simplices are

labelled by an element of $G$. We have a 2-simplex

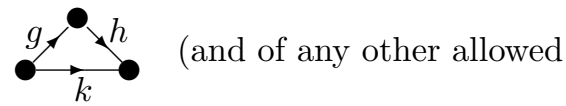
coloring) if the 0 -curvature condition is met, that is $g h=k$ in $G$. (No face index is needed.) The 3-simplices are built up from the 2 -simplices without any further restriction. A solution of the Big Pentagon equation can then be given by $F_{0}=F_{1}=F_{2}=F_{3}=F_{4} \equiv 1$ for any allowed set of the labels of the 1 -simplices. It is left to the reader to compute the corresponding structural maps of the weak $C^{*}$-Hopf algebra. 
The construction described above is clearly not the most general. It is easy to see, that it always leads to abelian intersection subalgebras $A^{L}$ and $A^{R}$. However, to any pair of finite dimensional weak $C^{*}$-Hopf algebras $A$ and $\hat{A}$ in duality, one can associate a (not necessarily unitary) solution of the Big Pentagon equation as follows:

The 0-simplices of the complex are $\bullet$ and $\bigcirc$. The 1-simplices of type

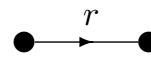

and $\bigcirc \stackrel{\hat{r}}{\longrightarrow}$ carry labels referring to the equivalence classes of the irreducible representations (simple direct summands $M_{n_{r}}(\mathbb{C})$ ) of $A$ and $\hat{A}$, respectively. There is only one 1-simplex of type labelled by $\alpha=1 \ldots N_{p q}^{r}$ where $N_{p q}^{r}$ denotes the fusion coefficients of the irreducible representations of $A$ and $\hat{A}$, respectively. The 2-simplices of type

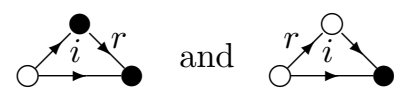
carry labels $i=1 \ldots n_{r}$, so that they serve as an orthonormal basis for the linear space carrying the defining representation of the simple direct summand $M_{n_{r}}(\mathbb{C})$ of $A$ and $\hat{A}$, respectively. We have all the 3 -simplices built up from these allowed 2 -simplices.

The 3-chains $F_{k}$ are then identified with the appropriate representation theoretical data: $F_{1}$ comprises the Wigner coefficients for $A, \bar{F}_{3}$ for $\hat{A} . F_{0}$ and $\bar{F}_{4}$ are obtained as the corresponding recoupling coefficients. $F_{2}$ is the Ocneanu cell read off the pairing between $A$ and $\hat{A}$.

This solution fails to be unitary in general. One can prove instead of unitarity only $F_{1}^{*} F_{1}=F_{3} F_{3}^{*}=\mathbb{1}$ (expressing the orthonormality of the Wigner coefficients) and the invertibility of $F_{2}$ in the sense of (3.4) (which is equivalent to the non-degeneracy of the pairing between $A$ and $\hat{A}$.)

Notice that the above association of a complex $K$ to $A$ and $\hat{A}$ is not unique. The fusion rules of $A$ and $\hat{A}$ determine the subcomplex generated by $\bullet$ and $\bigcirc$, respectively. The $C^{*}$-algebra structures of $A$ and $\hat{A}$ determine only the numbers of the 2 -simplices

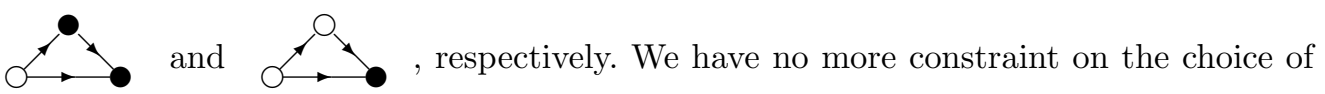
the complex.

As a last example we construct a finite dimensional weak $C^{*}$-Hopf algebra with nonAbelian intersection subalgebras which - since it is generated by $A^{L}$ and $A^{R}$ - can be considered as the blowing up of "nothing". It corresponds to a non-unitary solution of the Big Pentagon equation.

Let $N$ be any natural number and define the $C^{*}$-algebra $A:=M_{N}(\mathbb{C}) \otimes M_{N}(\mathbb{C})$. Choose matrix units $\left\{e_{i j}\right\}_{i j=1 \ldots N}$ in the first factor and $\left\{e^{i j}\right\}_{i j=1 \ldots N}$ in the second. Then

$$
\varepsilon\left(e_{i j} e^{k l}\right):=\frac{1}{N} \delta_{i k} \delta_{l j}
$$




$$
\begin{aligned}
& \Delta\left(e_{i j} e^{k l}\right):=\frac{1}{N} \sum_{x x^{\prime}} e_{x x^{\prime}} e^{k l} \otimes e_{i j} e^{x x^{\prime}} \\
& S\left(e_{i j} e^{k l}\right):=e_{l k} e^{j i}
\end{aligned}
$$

define a weak $C^{*}$-Hopf algebra in which the algebra generated by the elements $\left\{e_{i j}\right\}$ and $\left\{e^{i j}\right\}$ are $A^{R}$ and $A^{L}$, respectively.

This weak $C^{*}$-Hopf algebra corresponds to the solution of the Big Pentagon equation on the complex which has the two 0-simplices and $\bigcirc$, the 1-simplices

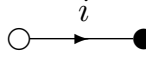

$i=1 \ldots N$ and the only 1 -simplices of type

We have all the allowed 2-simplices built up from these 1-simplices, without introducing any new face-label. Finally we have all the possible 3 -simplices. The 3 -chains $F_{k} k=0 \ldots 4$ are: $F_{0} \equiv 1, F_{1} \equiv \frac{1}{\sqrt{N}}, F_{2} \equiv \frac{1}{N}, F_{3} \equiv \frac{1}{\sqrt{N}}$ and $F_{4} \equiv 1$ for any allowed set of the labels on the 1 -simplices. One checks that $F$ solves the Big Pentagon equation and $F_{1}^{*} F_{1}=F_{3} F_{3}^{*}=\mathbb{1}$, but not the unitarity condition (3.3).

\section{References}

[1] G. Böhm, F. Nill, K. Szlachányi, Weak Hopf Algebras, in preparation.

[2] S. Baaj, G. Skandalis, Ann. Sci. ENS 26, 425 (1993).

[3] G. Böhm, K. Szlachányi, A Coassociative $C^{*}$-Quantum Group with Non-Integral Dimensions, q-alq/9509008, Lett. Math. Phys. 35, 437 (1996).

[4] S. Doplicher, J. E. Roberts, Ann. Math. 13075 (1989), and Commun. Math. Phys. 131 , 51 (1990).

[5] V. G. Drinfeld, Leningrad Math. J. 1 1419, (1990).

[6] K. Fredenhagen, K.-H. Rehren and B. Schroer, Commun. Math. Phys. 125, 201 (1989).

[7] Fuchs, Ganchev, Vecsernyés, Towards a classification of rational Hopf algebras, preprint NIKHEF-H/94-05 KL-TH-94/4, hep-th 9402153.

[8] R. Haag, Local Quantum Physics, Springer 1992.

[9] G. Mack and V. Schomerus, Endomorphisms and Quantum Symmetry of the Conformal Ising Model, in Algebraic Theory of Superselection Sectors, ed.: D. Kastler, World Scientific Singapore 1990.

[10] G. Mack and V. Schomerus, Nucl. Phys. B370, 185 (1992).

[11] F. Nill, K. Szlachányi, H.-W. Wiesbrock, in preparation.

[12] A. Ocneanu, Quantum Cohomology, Quantum Groupoids, and Subfactors, talk presented at the First Caribic School of Mathematics and Theoretical Physics, Guadeloupe 1993 (unpublished).

[13] K.-H. Rehren, Braid Group Statistics and their Superselection Rules in: Algebraic Theory of Superselection Sectors, ed. D. Kastler, World Scientific 1990.

[14] V. Schomerus, Quantum symmetry in quantum theory, DESY 93-018 preprint.

[15] M. E. Sweedler, Hopf algebras, Benjamin 1969.

[16] P. Vecsernyés, Nucl. Phys. B 415, 557 (1994).

[17] S. L. Woronowicz, Commun. Math. Phys. 111, 613 (1987). 